\title{
QUANTUM GRAVITATIONAL COLLAPSE IN THE LEMAITRE-TOLMAN-BONDI MODEL WITH A POSITIVE COSMOLOGICAL CONSTANT
}

\author{
Anne Franzen 1 \\ Institute for Theoretical Physics, \\ University of Cologne, \\ Zülpicher Strasse 77, 50937 Köln, Germany \\ Sashideep Gutt:2 \\ The Harish-Chandra Research Institute, \\ Chhatnag Road, Jhunsi, Allahabad, \\ Uttar Pradesh 211019, India

\section{Claus Kiefer} \\ Institute for Theoretical Physics, \\ University of Cologne, \\ Zülpicher Strasse 77, 50937 Köln, Germany.
}

\begin{abstract}
Previous papers dealt with the quantization of the Lemaitre-Tolman-Bondi (LTB) model for vanishing cosmological constant $\Lambda$. Here we extend the analysis to the case $\Lambda>0$. Our main goal is to present solutions of the Wheeler-DeWitt equation, to give their interpretation, and to derive Hawking radiation from them. We restrict ourselves to a discussion of those points that are different from the $\Lambda=0$-case. These have mainly to do with the occurrence of two horizons.
\end{abstract}

\footnotetext{
${ }^{1}$ New address from June 1, 2009: Institute for Theoretical Physics, Utrecht University, Leuvenlaan 4, 3584 CE Utrecht, The Netherlands

${ }^{2}$ Part of the work was completed before August 2008 at Tata Institute of Fundamental Research, Homi Bhabha Road, Mumbai, 400005, India.
} 


\section{Introduction}

Part of the research on quantum gravity consists in the attempt to get a possible insight into the final theory from concrete models. The simplest models deal with spatially homogeneous cosmological metrics and are typically used in quantum cosmology. Less simple are models with spherical symmetry. They are used in cosmology, too, but mainly in the description of black holes.

Here we deal with a particular spherically-symmetric model, the Lemaitre-Tolman-Bondi (LTB) model. It describes self-gravitating inhomogeneous dust and is well understood at the classical level where it is mostly used in cosmology [1]. The quantization of this model was attempted in both quantum geometrodynamics and loop quantum gravity. Here we restrict ourselves to quantum geometrodynamics, in which the central equations are the Wheeler-DeWitt equation and the momentum (diffeomorphism) constraints [2, 3].

Following the application of canonical geometrodynamics to the Schwarzschild black hole [4] and to Reissner-Nordström-anti-de Sitter black holes [5], the canonical formalism for the LTB model was developed in [6] and then applied to quantization in a series of papers, see [7, 8, 9] and the references therein. While it was not possible to construct the quantum theory for the LTB model in a rigorous way, partial progress was achieved. Among the results was the recovery of Hawking radiation plus greybody corrections from exact solutions to the Wheeler-DeWitt equation and the momentum constraints [8, 9]. They were found for the case of vanishing cosmological constant $\Lambda$ where only one horizon (the black-hole horizon) is present. Here we extend the analysis to the case $\Lambda>0$, where two horizons (the blackhole and the cosmological horizon) exist. We again manage to find exact quantum states from which the two different Hawking temperatures from the two horizons can be recovered.

Although we are mainly interested in understanding quantum gravity, we want to emphasize that the case $\Lambda>0$ fits very well current cosmological observations [10]. The analysis for $\Lambda<0$, where only one horizon is present, was performed in [11].

In our present paper we shall focus on the main differences to the case of vanishing $\Lambda$. For more details on the general formalism we refer to the earlier papers cited above. The LTB model was also addressed from the perspective of loop quantum gravity [12]. 


\section{The classical LTB model with positive cosmo- logical constant}

Since we deal with dust, the energy-momentum tensor is given by $T_{\mu \nu}=$ $\epsilon(\tau, \rho) u_{\mu} u_{\nu}$, where $u^{\mu}=u^{\mu}(\tau, \rho)$ is the four-velocity vector of a dust particle with proper time $\tau$; the parameter $\rho$ labels the various shells which together form the dust cloud. The line element for the LTB spacetime in comoving synchronous coordinates is given by

$$
\mathrm{d} s^{2}=-\mathrm{d} \tau^{2}+\frac{\left(\partial_{\rho} R\right)^{2}}{1+2 E(\rho)} \mathrm{d} \rho^{2}+R^{2}(\rho, \tau)\left(\mathrm{d} \theta^{2}+\sin ^{2} \theta \mathrm{d} \phi^{2}\right) .
$$

Inserting this expression into the Einstein field equations leads to

$$
8 \pi G \epsilon(\tau, \rho)=\frac{\partial_{\rho} F}{R^{2} \partial_{\rho} R}
$$

and

$$
\left(\partial_{\tau} R\right)^{2}=\frac{F}{R}+\frac{\Lambda R^{2}}{3}+2 E \equiv 1-\mathcal{F}+2 E,
$$

where $F(\rho) \equiv 2 G M(\rho)$ is a non-negative function with the dimension of a length, and

$$
\mathcal{F} \equiv 1-\frac{F}{R}-\frac{\Lambda R^{2}}{3}
$$

The case of collapse is described by $\partial_{\tau} R(\tau, \rho)<0$. We set $c=1$ throughout.

There still exists the freedom to rescale the shell index $\rho$. This freedom can be removed by demanding

$$
R(0, \rho)=\rho,
$$

so that for $\tau=0$ the label coordinate $\rho$ is equal to the curvature radius $R$. Now we can express the functions $F(\rho)$ and $E(\rho)$ in terms of the energy density $\epsilon$ at $\tau=0$. From (2) and (3) one gets

$$
\begin{aligned}
& F(\rho)=8 \pi G \int_{0}^{\rho} \epsilon(0, \tilde{\rho}) \tilde{\rho}^{2} \mathrm{~d} \tilde{\rho}, \\
& E(\rho)=\frac{1}{2}\left[\partial_{\tau} R(\tau=0, \rho)\right]^{2}-\frac{F(\rho)}{2 \rho}-\frac{\Lambda \rho^{2}}{6} .
\end{aligned}
$$

The interpretation of these quantities is that $F(\rho) / 2 G \equiv M(\rho)$ is the active gravitating mass within a $\rho=$ constant shell, while $E(\rho)$ is the total energy per unit mass within the same shell. The marginally bound models are defined by $E(\rho) \equiv 0$. Here we consider the general case, which includes the non-marginal case defined by $E(\rho) \neq 0$. 
In order to derive Hawking radiation, we shall consider the following situation. We assume the presence of a black hole with mass $M_{0}$ surrounded by a gravitating dust cloud. We assume, moreover, that the total energy of the cloud is small compared to the mass of the black hole. After quantization, we shall find exact quantum states for the dust cloud from which the Hawking temperatures will be found. The dust cloud will play the role of the quantum fields usually employed in the derivation of Hawking radiation.

If the dust cloud is of finite extension, the metric outside of it is of the Schwarzschild-de Sitter (SdS) or Kottler form 3

$$
\begin{aligned}
\mathrm{d} s^{2}= & -\left(1-\frac{2 G M}{R}-\frac{\Lambda R^{2}}{3}\right) \mathrm{d} T^{2} \\
& +\left(1-\frac{2 G M}{R}-\frac{\Lambda R^{2}}{3}\right)^{-1} \mathrm{~d} R^{2}+R^{2} \mathrm{~d} \Omega^{2},
\end{aligned}
$$

where $M$ is the mass of the black hole plus the mass contribution of the cloud. For small $R$, SdS space approximates Schwarzschild space, while for large $R$ and positive $\Lambda$ it approximates de Sitter space. The black-hole horizon $R_{\mathrm{h}}$ and the cosmological horizon $R_{\mathrm{c}}$ are two of the three zeros of $g_{T T}$ in (8), see, for example, [14] and [15]. They are explicitly given by

$$
\begin{aligned}
& R_{\mathrm{h}}=3 \operatorname{GM\ell \xi }\left(1-\sqrt{1-\frac{1}{\ell \xi^{3}}}\right)=\frac{\xi}{\sqrt{\Lambda}}\left(1-\sqrt{1-\frac{1}{\ell \xi^{3}}}\right), \\
& R_{\mathrm{c}}=3 \operatorname{GM} \ell \xi\left(1+\sqrt{1-\frac{1}{\ell \xi^{3}}}\right)=\frac{\xi}{\sqrt{\Lambda}}\left(1+\sqrt{1-\frac{1}{\ell \xi^{3}}}\right),
\end{aligned}
$$

where $\ell^{-1}=3 G M \sqrt{\Lambda}$ and $\xi=\cos \left(\frac{1}{3} \cos ^{-1}\left[\ell^{-1}\right]\right)$. The third zero value, $R_{\mathrm{n}}$, is negative, $R_{\mathrm{n}}=-R_{\mathrm{h}}-R_{\mathrm{c}}=-6 G M \ell \xi$ and possesses no obvious meaning. 4 The LTB solution (11) must then be matched at the dust boundary to the SdS solution.

In the canonical formalism, the general ansatz for a spherically-symmetric line element reads

$$
\mathrm{d} s^{2}=-N^{2} \mathrm{~d} t^{2}+L^{2}\left(\mathrm{~d} r-N^{r} \mathrm{~d} t\right)^{2}+R^{2} \mathrm{~d} \Omega^{2},
$$

where $N$ is the lapse function, $N^{r}$ is the only component of the shift vector that survives the symmetry reduction, and $\mathrm{d} \Omega^{2}=\mathrm{d} \theta^{2}+\sin ^{2} \theta \mathrm{d} \phi^{2}$ is the line element on the unit two-sphere.

\footnotetext{
${ }^{3}$ See, for example, [13 for a detailed discussion of this metric.

${ }^{4}$ It must be assumed here that the black-hole horizon is smaller than the cosmological horizon, $R_{\mathrm{h}}<R_{\mathrm{c}}$, which means that $\ell^{-1}<1$. This is well satisfied in our Universe: Inserting for $M$ the mass of the supermassive black hole in the quasar OJ287, which is presently the biggest known supermassive black hole, with $M \approx 1.8 \times 10^{10} M_{\odot}[16$, and for $\Lambda$ the value following from $\Omega_{\Lambda} \approx 0.726$ [10, we get $\ell^{-1} \approx 9 \times 10^{-11}$. The value $\ell=1$ would correspond to the Nariai metric for which both areas are the same but are separated by a finite proper distance. (See 17] for a discussion of the Nariai metric and a reprint of Nariai's original papers.)
} 
Inserting the ansatz (10) into the ADM form of the Einstein-Hilbert action, we obtain the gravitational part of the action,

$$
S^{\mathrm{g}}=\int \mathrm{d} t \int_{0}^{\infty} \mathrm{d} r\left(P_{L} \dot{L}+P_{R} \dot{R}-N \mathcal{H}^{\mathrm{g}}-N^{r} \mathcal{H}_{r}^{\mathrm{g}}\right)+S_{\partial \Sigma},
$$

where the Hamiltonian and the diffeomorphism (momentum) constraint are given by

$$
\begin{aligned}
& \mathcal{H}^{\mathrm{g}}=-G\left(\frac{P_{L} P_{R}}{R}-\frac{L P_{L}^{2}}{2 R^{2}}\right)+\frac{1}{G}\left[-\frac{L}{2}-\frac{R^{\prime 2}}{2 L}+\left(\frac{R R^{\prime}}{L}\right)^{\prime}+\frac{\Lambda R^{2} L}{2}\right], \\
& \mathcal{H}_{r}^{\mathrm{g}}=R^{\prime} P_{R}-L P_{L}^{\prime},
\end{aligned}
$$

respectively (a prime denotes a derivative with respect to $r$ ). The boundary action $S_{\partial \Sigma}$ will be discussed below.

The total action is the sum of (11) and an action $S^{\text {d }}$ describing the dust. The canonical formalism for the latter was developed in [18. The dust action reads

$$
S^{\mathrm{d}}=\int \mathrm{d} t \int_{0}^{\infty} \mathrm{d} r\left(P_{\tau} \dot{\tau}-N \mathcal{H}^{\mathrm{d}}-N^{r} \mathcal{H}_{r}^{\mathrm{d}}\right)
$$

where the contributions to the Hamiltonian and momentum constraints are

$$
\mathcal{H}^{\mathrm{d}}=P_{\tau} \sqrt{1+\frac{\tau^{\prime 2}}{L^{2}}} \text { and } \mathcal{H}_{r}^{\mathrm{d}}=\tau^{\prime} P_{\tau} .
$$

It is due to this relatively simple form of the dust action that exact quantum states can be found below. The full constraints are then the sum of the gravitational and the matter (dust) constraints.

The general ansatz of the metric (10) should, of course, correspond to the LTB metric (11). This leads to a couple of relations that are analogous to those derived in [7, except that $\mathcal{F}$ is now given by (4). One can express, in particular, the variables $E, F$, and $\tau$, which completely characterize the collapse of the dust cloud, in terms of the canonical variables.

What about the boundary action $S_{\partial \Sigma}$ in (11)? In principle, we have to consider two boundaries. The first boundary is at the centre of symmetry for the dust cloud, the second either at spatial infinity or at another chosen end of the spatial hypersurfaces. As for the inner boundary, the effect of a positive cosmological constant compared to a vanishing or negative $\Lambda$ is there negligible, because its effects are felt only at cosmological distances. We can thus employ the boundary action used previously, cf. [9]. It was found that the following term must be added to compensate a corresponding term that arises from variation of the action and partial integration:

$$
S_{\partial \Sigma}=\int_{\partial \Sigma} \mathrm{d} t N_{0}(t) M_{0}(t)
$$


where $N_{0}$ is the lapse function at $r=0$, and $M_{0}=F(0) / 2 G$ is the mass of the black hole at the centre. If this compensation were not made, one would conclude that a variation of $N_{0}$ leads to vanishing black-hole mass, which would certainly not be desirable. Such arguments were already used in the geometrodynamics of the pure Schwarzschild black hole [4].

The choice of the second boundary is less obvious. Since the spacetime here is asymptotically de Sitter, the spatial sections are no longer asymptotically flat. This leads basically to two options. One option is to choose a spacelike hypersurface that approaches the spacelike infinity in SdS spacetime. Here one must be careful and avoid $r$ to become timelike. This is, for example, achieved if we employ coordinates such as the Painlevé-Gullstrand coordinates used in [19] which are, in fact, close to the coordinates that we use below to simplify the constraints. Another option is to use the cosmological horizon as the second boundary. This would correspond to the choice frequently made in black-hole papers where the bifurcation sphere of the horizon is employed as the inner boundary, see, for example, [5] and [20]. It will also be our choice here.

We thus consider the case where the cosmological horizon is the second boundary. The situation is then analogous to the cases considered in [5] and [20]. We choose $r=0$ to represent the centre of the cloud and let $r \rightarrow \infty$ at the cosmological horizon. This is possible if we smoothly match $r$ to the tortoise coordinate $R_{*}$, defined by $\mathrm{d} R_{*}=\mathcal{F}^{-1} \mathrm{~d} r$, at the cosmological horizon. The time parameter $t$ is chosen equal to the Killing time $T$. Let us now turn to the fall-off conditions. As for $L$, we choose

$$
L(t, r)=\frac{L_{0}(t)}{r^{3}}+O\left(\frac{1}{r^{4}}\right) .
$$

$L$ tends to zero near the cosmological horizon, where $r$ goes to infinity. This is required, since it is equal to the tortoise coordinate there. It is proportional to $1 / r^{3}$, which guarantees that the distance to the horizon will be finite. As for $R$, we choose

$$
R(t, r)=R_{\mathrm{c}}-\frac{R_{1}}{r^{2}}+\frac{R_{2}(t)}{r^{3}}+O\left(\frac{1}{r^{4}}\right) .
$$

For the remaining variables we impose

$$
N(t, r)=\frac{N_{0}(t)}{r^{2}}+O\left(\frac{1}{r^{3}}\right), \quad N^{r}=O\left(\frac{1}{r}\right),
$$

and

$$
P_{L}=O\left(\frac{1}{r}\right), \quad P_{R}=O\left(\frac{1}{r}\right) .
$$

These fall-offs are consistent with the equations of motion and the constraint. They also guarantee that the boundary term at the cosmological horizon vanishes. 
One could now start with the quantization of (12), (13), and (14). However, this turns out to be too complicated. One thus performs first a classical simplification of the constraints in order to render them manageable. This is achieved by introducing new variables and appropriate canonical transformations [6, 7]. In this way, the variable $\Gamma \equiv F^{\prime}$ and its momentum $P_{\Gamma}$ are introduced as new canonical variables; this choice is also convenient because one thereby absorbs a certain boundary term [7]. Using then the momentum constraints to eliminate $P_{\Gamma}$ and squaring the ensuing Hamilton constraint, one arrives at the following final form of the constraints: The full Hamiltonian constraint reads

$$
\mathcal{H}=P_{\tau}^{2}+\mathcal{F} P_{R}^{2}-\frac{\Gamma^{2}}{4 G^{2} \mathcal{F}} \approx 0,
$$

while the full momentum constraint reads

$$
\mathcal{H}_{r}=R^{\prime} P_{R}-\Gamma P_{\Gamma}^{\prime}+\tau^{\prime} P_{\tau} \approx 0 .
$$

This is the form of the constraints suitable for quantization. Strictly speaking, re-defining the constraints is part of the very definition of quantization. There are many cases where the classical constraints are transformed into a manageable form by canonical transformations, see, for example, [21.

We note that because of the involved squaring the new Hamiltonian constraint (21) has acquired the dimension (mass/length) ${ }^{2}$. We emphasize that the only differences compared to the earlier papers dealing with non-positive $\Lambda$ lie so far in the definition of $\mathcal{F}$ according to (44). We also emphasize that through these manipulations the kinetic term in the Hamiltonian constraint is no longer hyperbolic, in contrast to the original form (12). More precisely, it is hyperbolic inside the black-hole horizon and outside the cosmological horizon, and it is elliptic between the horizons 5 A similar observation was made for the Reissner-Nordström black hole already in [22, 6] This gives further support for choosing a hypersurface that extends from one horizon to the other. We finally note that the gravitational constant $G$ occurs in (21) in the same way as in the usual Hamiltonian constraint without matter; this may be of relevance for semiclassical approximation schemes in the quantum theory [2].

The SdS spacetime is static only in between the two horizons. It thus makes sense to talk about a Killing time, $T$, only in this region. In previous

\footnotetext{
${ }^{5}$ One might think that this change of sign is related to the fact that the metric in the interior of the horizon can become "cosmological", as it happens e.g. for the Schwarzschild black hole where the inside metric is of the Kantowski-Sachs form. This is, however, not the case. The equation $\mathcal{F}=0$ is a condition for an apparent horizon, and the sign change happens also for the non-static case where the interior is not "cosmological".

${ }^{6} \mathrm{It}$ is interesting to note that the Einstein equations for regular axisymmetric and stationary black holes surrounded by matter are also elliptic in the exterior and hyperbolic in the interior of the hole [23].
} 
papers it was shown that $T=2 P_{\Gamma}$ and that

$$
T=a \tau \pm \int \mathrm{d} R \frac{\sqrt{1-\mathcal{F} a^{2}}}{\mathcal{F}}
$$

where $a \equiv 1 / \sqrt{1+2 E}$; the plus sign in (23) holds for an expanding, the minus sign for a collapsing dust cloud. (This interpretation comes from the similarity of the coordinates $(\tau, R)$ with the Painlevé-Gullstrand coordinates for a Schwarzschild black hole [24].) It is straightforward to show that the same relation also holds in our case, taking into account the new definition for $\mathcal{F}$. Strictly speaking, one can talk about a Killing time only in the absence of the dust cloud, where the spacetime is of SdS form. However, as in the previous papers, one can approximately continue to interpret $T$ as a Killing time, because we consider the dust only as a small perturbation to the SdS spacetime.

\section{Quantization}

We now turn to quantization. Applying the formal Dirac procedure, we replace the momenta by functional derivatives with respect to the corresponding configuration variables. From the Hamiltonian constraint (21) we get the Wheeler-DeWitt equation,

$$
\begin{aligned}
{\left[-G^{2} \hbar^{2}\left(\frac{\delta^{2}}{\delta \tau(r)^{2}}\right.\right.} & +\mathcal{F} \frac{\delta^{2}}{\delta R(r)^{2}}+A(R, F) \delta(0) \frac{\delta}{\delta R(r)} \\
& \left.\left.+B(R, F) \delta(0)^{2}\right)-\frac{\Gamma^{2}}{4 \mathcal{F}}\right] \Psi[\tau(r), R(r), \Gamma(r)]=0 .
\end{aligned}
$$

Here, $A$ and $B$ are smooth functions of $R$ and $F$, which encapsulate the factor-ordering ambiguities. The factor-ordering problem is a fundamental problem that can only be dealt with after a suitable regularization procedure has been invoked. A general treatment is beyond the scope of our paper. To implement the factor-ordering ambiguities at a formal level, we have introduced formal factors of $\delta(0)$ into the equation. The reason is that the equation can then be put onto a lattice and the continuum limit can be performed [7]. This corresponds to a particular regularization procedure that allows the presentation of the solutions below.

From (22) one gets the quantum momentum constraint

$$
\left[\tau^{\prime} \frac{\delta}{\delta \tau(r)}+R^{\prime} \frac{\delta}{\delta R(r)}-\Gamma\left(\frac{\delta}{\delta \Gamma(r)}\right)^{\prime}\right] \Psi[\tau(r), R(r), \Gamma(r)]=0 .
$$

Both equations, (24) and (25), can be put on the lattice and a special class of exact solutions can be found [7]. In the continuum limit, this class of 
solutions reads

$$
\begin{aligned}
& \Psi[\tau(r), R(r), \Gamma(r)]= \\
& \exp \left( \pm \frac{\mathrm{i}}{2 G \hbar} \int \mathrm{d} r \Gamma\left[a \tau+\int^{R} \mathrm{~d} R \frac{\sqrt{1-a^{2} \mathcal{F}}}{\mathcal{F}}\right]\right) .
\end{aligned}
$$

(In the earlier papers, a more general solution with an unspecified constant $b$ was used [7]; for simplicity, we set here $b=0$.) The momentum constraint (25) is solved by any state of the form $\exp \left(\mathrm{i} /(2 G \hbar) \int \mathrm{d} r \Gamma W\right)$, where $W$ is a smooth function of $R$ and $\tau$. The Wheeler-DeWitt equation, however, is only solved for a particular factor ordering. Generalizing the treatment presented in [7] to $\Lambda \neq 0$, one must choose in (24) $B=0$ and

$$
A=\frac{1}{2}\left(\frac{F}{R^{2}}-\frac{2 \Lambda R}{3}\right) \frac{2-a^{2} \mathcal{F}}{1-a^{2} \mathcal{F}} .
$$

This means that $\Lambda$ does not only enter the definition of $\mathcal{F}$, but also the chosen factor ordering. Exact solutions for the quantum states can only be found for this special $\Lambda$-dependent choice of $A$. This is certainly a weak point of our approach, because there should be a fixed factor ordering at the fundamental level. Nevertheless, this choice for $A$ gives us the opportunity to have exact solutions at our disposal. Moreover, at the semiclassical level of WKB states, the factor ordering is irrelevant, and the calculation of Hawking radiation below is an effect that occurs at the WKB level [25]. In fact, one can use a semiclassical expansion scheme instead of the exact solutions to derive Hawking radiation and quantum gravitational corrections [26].

\section{Hawking radiation}

Let us now turn to the calculation of Hawking radiation. The idea is to remain as close as possible to Hawking's original derivation in [27, but to transfer this idea to solutions of the Wheeler-DeWitt equation and the momentum constraints, which are full quantum gravitational states. Because the Wheeler-DeWitt equation (24) contains second derivatives with respect to the dust time $\tau$ (which is a consequence of squaring the Hamiltonian at the classical level), we can define states of positive and negative frequency with respect to $\tau$. This is crucial for our discussion. The situation is thus analogous to the Klein-Gordon equation, where positive frequency is defined by a minus sign in the phase, $\exp (-\mathrm{i} E t / \hbar)$, and correspondingly negative frequency by a positive sign.

For the state with positive frequency we thus get from (26),

$$
\begin{aligned}
& \Psi^{+}[\tau(r), R(r), \Gamma(r)]= \\
& \exp \left(-\frac{\mathrm{i}}{2 G \hbar} \int \mathrm{d} r \Gamma\left[a \tau+\int^{R} \mathrm{~d} R \frac{\sqrt{1-a^{2} \mathcal{F}}}{\mathcal{F}}\right]\right),
\end{aligned}
$$


while for the state with negative energy we have

$$
\begin{aligned}
& \Psi^{-}[\tau(r), R(r), \Gamma(r)]= \\
& \exp \left(\frac{\mathrm{i}}{2 G \hbar} \int \mathrm{d} r \Gamma\left[a \tau+\int^{R} \mathrm{~d} R \frac{\sqrt{1-a^{2} \mathcal{F}}}{\mathcal{F}}\right]\right) .
\end{aligned}
$$

In Hawking's calculation in [27, the Bogolyubov coefficient $\beta$, which gives the negative-frequency part of the time-developed original state with positive frequency, plays a central role. Here, the role of the quantum matter field in [27] is played by the dust. We thus define Hawking radiation for the black-hole case as the overlap between an "outgoing dust state" with negative energy and an "ingoing dust state" with positive energy. Since the interpretation of these states is made with respect to an observer in the SdS spacetime using the Killing time $T$, we have to substitute the dust time by $T$ according to (23). Taking the corresponding signs in (23) into account, we have then to calculate the overlap between

$$
\Psi_{\mathrm{e}}^{-}=\exp \left(\frac{\mathrm{i}}{2 G \hbar} \int \mathrm{d} r \Gamma T\right),
$$

which is actually independent of $R$, and

$$
\Psi_{\mathrm{c}}^{+}=\exp \left(-\frac{\mathrm{i}}{2 G \hbar} \int \mathrm{d} r \Gamma T-\frac{\mathrm{i}}{G \hbar} \int \mathrm{d} r \Gamma \int^{R} \mathrm{~d} R \frac{\sqrt{1-a^{2} \mathcal{F}}}{\mathcal{F}}\right),
$$

where the index e(c) denotes "expanding" ("collapsing").

For the cosmological horizon, the situation is just the opposite because the Hawking radiation is now incoming from the horizon. We thus have to calculate the overlap between the "ingoing negative-energy state"

$$
\Psi_{\mathrm{c}}^{-}=\exp \left(\frac{\mathrm{i}}{2 G \hbar} \int \mathrm{d} r \Gamma T+\frac{\mathrm{i}}{G \hbar} \int \mathrm{d} r \Gamma \int^{R} \mathrm{~d} R \frac{\sqrt{1-a^{2} \mathcal{F}}}{\mathcal{F}}\right)
$$

and the "outgoing positive-energy state"

$$
\Psi_{\mathrm{e}}^{+}=\exp \left(-\frac{\mathrm{i}}{2 G \hbar} \int \mathrm{d} r \Gamma T\right) .
$$

In order to calculate the overlap between the quantum states (30) and (31), or between (32) and (33), we need a Hilbert space. As far as full quantum gravity is concerned, this is an open issue [2]. In the present case, however, a natural candidate is present [25, 8]. We can choose the standard Schrödinger inner product with respect to constant time and $R$ as the integration variable. The integration range is supposed to run from the black-hole horizon, $R_{\mathrm{h}}$, to the cosmological horizon, $R_{\mathrm{c}}$. This is further 
motivated by the fact that our hypersurface lies between the horizons; as we have already noted, in this region the kinetic term in (21) is elliptic.

Addressing first the cosmological horizon, we have for the desired overlap between ingoing negative-energy and outgoing positive-energy states the expression

$$
\left\langle\Psi_{\mathrm{c}}^{-} \mid \Psi_{\mathrm{e}}^{+}\right\rangle \equiv \int_{R_{\mathrm{h}}}^{R_{\mathrm{c}}} \mathrm{d} R \sqrt{g_{R R}} \Psi_{\mathrm{c}}^{-*} \Psi_{\mathrm{e}}^{+} .
$$

Here, $g_{R R}$ is the $R R$-component of the DeWitt metric, as it can be read off (21) where its inverse is the prefactor of the term $P_{R}^{2}$, and we thus have $g_{R R}=\mathcal{F}^{-1} \overline{7}$

There is, however, one further point to consider. Since we are interested in an observer far away from the dust cloud, for whom the Killing time $T$ according to (23) is the appropriate time coordinate, we have already rewritten the states in terms of $T$. Consequently, as in [25, 8], we have to evaluate also the component $g_{R R}$ in the new coordinate system $(T, R)$ instead of $(\tau, R)$. Using (23), one gets $\sqrt{g_{R R}}=(a \mathcal{F})^{-1}$. This is the expression to be used in the calculation of the Bogolyubov coefficient.

For the concrete calculation we shall write the full states as a product of single-shell states where the radial variable $r$ is assumed to consist of discrete points separated by a distance $\sigma$. (The continuum limit is obtained for $\sigma \rightarrow 0$.) As in [8], the Bogolyubov coefficient $\beta$ is calculated for each shell separately. In the discrete case, we replace $\Gamma$ by the dimensionless variable $2 \omega$ and indicate the dependence on $\omega$ by an index. (The factor 2 is motivated by the fact that $\Gamma=2 M^{\prime}$.) We omit the shell index and write the corresponding wave functions as $\psi_{\omega}(T, R)$. From (34) we then define $\beta$ to read

$$
\beta_{\omega \omega^{\prime}} \equiv \int_{R_{\mathrm{h}}}^{R_{\mathrm{c}}} \mathrm{d} R \sqrt{g_{R R}} \Psi_{\mathrm{c} \omega}^{-*} \Psi_{\mathrm{e} \omega^{\prime}}^{+} .
$$

In the earlier papers [8] and [9] we have chosen a particular normalization for $\beta$, which was motivated by the normalization of states for the Klein-Gordon equation. Since the full normalization of solutions to the Wheeler-DeWitt equation is anyway not known, we shall leave the question of normalization open here and define $\beta$ directly by (35).

Employing now the one-shell contributions of (32) and (33) and inserting

\footnotetext{
${ }^{7}$ Note that this measure is not the measure that would render the Wheeler-DeWitt equation (24) with the factor ordering (27) Hermitean 9. Our choice of the measure in (34) would make the $R$-part of (24) Hermitean if the Laplace-Beltrami factor ordering were used.
} 
them into (35), one gets the following expression:

$$
\begin{aligned}
\beta_{\omega \omega^{\prime}}= & \sqrt{1+2 E} \exp \left(-\frac{\mathrm{i} \sigma T}{G \hbar}\left[\omega+\omega^{\prime}\right]\right) \times \\
& \int_{R_{\mathrm{h}}}^{R_{\mathrm{c}}} \mathrm{d} R \mathcal{F}^{-1} \exp \left(-\frac{2 \mathrm{i} \sigma \omega}{G \hbar} \int^{R} \mathrm{~d} R \frac{\sqrt{1-a^{2} \mathcal{F}}}{\mathcal{F}}\right) .
\end{aligned}
$$

We shall first calculate the contribution to $\beta_{\omega \omega^{\prime}}$ from the cosmological horizon. This will be achieved through an appropriate near-horizon approximation, making the standard assumption that the integral is dominated by the close vicinity of the horizon. We start by writing $\mathcal{F}^{-1}$ in terms of partial fractions:

$$
\begin{aligned}
\frac{1}{\mathcal{F}} & =\frac{1}{1-\frac{F}{R}-\frac{\Lambda R^{2}}{3}} \\
& =R\left[\frac{A}{R-R_{\mathrm{c}}}+\frac{B}{R-R_{\mathrm{h}}}+\frac{C}{R-R_{\mathrm{n}}}\right] .
\end{aligned}
$$

Now we introduce the variable $s$ by $l s=R_{\mathrm{c}}-R$ so that near the cosmological horizon $|s| \ll 1$. Here, $l \equiv 1 / \sqrt{\Lambda}$.

First we consider the term

$$
\begin{aligned}
\int^{R} \mathrm{~d} R \frac{\sqrt{1-a^{2} \mathcal{F}}}{\mathcal{F}}= & \int^{s}-l \mathrm{~d} s\left(R_{\mathrm{c}}-l s\right)\left[-\frac{A}{l s}+\frac{B}{R_{\mathrm{c}}-R_{\mathrm{h}}-l s}+\frac{C}{R_{\mathrm{c}}-R_{\mathrm{n}}-l s}\right] \\
& \times \sqrt{1-\frac{a^{2}}{R_{\mathrm{c}}-l s}\left(\frac{1}{-\frac{A}{l s}+\frac{B}{R_{\mathrm{c}}-R_{\mathrm{h}}-l s}+\frac{C}{R_{\mathrm{c}}-R_{\mathrm{n}}-l s}}\right)}
\end{aligned}
$$

After some calculation, neglecting terms with a positive power in $s$, we get:

$$
\int^{R} \mathrm{~d} R \frac{\sqrt{1-a^{2} \mathcal{F}}}{\mathcal{F}} \approx \int^{s} \mathrm{~d} s \sqrt{\text { const }-\frac{1}{s} \xi+\frac{1}{s^{2}} A^{2} R_{\mathrm{c}}^{2}}
$$

with $\xi=2 A^{2} l R_{\mathrm{c}}+\frac{2 A B R_{\mathrm{c}}^{2} l}{R_{\mathrm{c}}-R_{\mathrm{h}}}+\frac{2 A C R_{\mathrm{c}}^{2} l}{R_{\mathrm{c}}-R_{\mathrm{n}}}-a^{2} A l R_{\mathrm{c}}$. Expanding (39) for $|s| \ll 1$ yields

$$
\begin{aligned}
\int^{s} \mathrm{~d} s \sqrt{\text { const }-\frac{1}{s} \xi+\frac{1}{s^{2}} A^{2} R_{c}^{2}} & \approx \int^{s} \mathrm{~d} s \frac{1}{s}\left[A R_{c}-\frac{\xi s}{2 A R_{c}}+\mathcal{O}\left(s^{2}\right)\right] \\
& \approx A R_{c} \ln |s|-\frac{\xi s}{2 A R_{c}}
\end{aligned}
$$

Inserting this in our integral (36) and using

$$
\mathcal{F}^{-1} \approx \frac{A R}{R-R_{\mathrm{c}}} \approx-\frac{A R_{\mathrm{c}}}{l s}
$$


we obtain

$$
\begin{aligned}
\beta_{\omega \omega^{\prime}}= & -\sqrt{1+2 E} \exp \left(-\mathrm{i} \frac{\sigma T}{G \hbar}\left(\omega+\omega^{\prime}\right)\right) A R_{\mathrm{c}} \\
& \times \int_{0}^{\left(R_{\mathrm{c}}-R_{\mathrm{h}}\right) / l} \mathrm{~d} s s^{-2 \mathrm{i} \sigma \omega A R_{\mathrm{c}} / G \hbar-1} \exp \left[\frac{\mathrm{i} \xi}{A R_{\mathrm{c}}} \frac{\sigma \omega s}{G \hbar}\right] .
\end{aligned}
$$

To evaluate this integral, we use the formula 28

$$
\int_{0}^{\infty} \mathrm{d} x x^{\nu-1} e^{-(p+\mathrm{i} q) x}=\Gamma(\nu)\left(p^{2}+q^{2}\right)^{-\nu / 2} e^{-\mathrm{i} \nu \arctan (q / p)}
$$

which is, in particular, applicable to the case $p=0$ and $0<\operatorname{Re} \nu<1$. (We insert a small positive value for Re $\nu$, which we let go to zero after the integration.) Then,

$$
\begin{aligned}
& \beta_{\omega \omega^{\prime}}=-\sqrt{1+2 E} \exp \left(-\mathrm{i} \frac{\sigma T}{G \hbar}\left(\omega+\omega^{\prime}\right)\right) A R_{\mathrm{c}} \times \\
& \Gamma\left(-\frac{2 \mathrm{i} \sigma \omega A R_{\mathrm{c}}}{G \hbar}\right)\left(-\frac{\sigma \omega \xi}{G \hbar A R_{\mathrm{c}}}\right)^{2 \mathrm{i} \sigma \omega A R_{\mathrm{c}} / G \hbar} e^{\pi \sigma \omega A R_{\mathrm{c}} / G \hbar} .
\end{aligned}
$$

Using

$$
\Gamma(-\mathrm{i} y) \Gamma(\mathrm{i} y)=\frac{\pi}{y \sinh \pi y}
$$

(with real $y$ ), we get

$$
\left|\beta_{\omega \omega^{\prime}}\right|^{2}=-\frac{\pi G \hbar A R_{\mathrm{c}}(1+2 E)}{\sigma \omega} \frac{1}{e^{-4 \pi \sigma \omega A R_{c} / G \hbar}-1}
$$

for the absolute square of $\beta$. (In spite of the minus sign, this is positive because $A<0$, see below.) Next we want to calculate the particle creation rate, which means we have to evaluate the expression $\Sigma_{k} \beta_{i k} \beta_{i k}^{*}$. Here this corresponds to the integral $\int_{0}^{\infty} \mathrm{d} \omega^{\prime}\left|\beta_{\omega \omega^{\prime}}\right|^{2}$. This integral diverges and hence needs to be suitably regulated. We introduce a decay factor $e^{-b \omega^{\prime}}$ and carry out the integration. For the "in" particle number operator we then obtain

$$
\left\langle\text { in }\left|\hat{N}_{\text {in }}\right| \text { in }\right\rangle=-\frac{\pi G \hbar A R_{\mathrm{c}}(1+2 E)}{b \sigma \omega} \frac{1}{e^{-4 \pi \sigma \omega A R_{c} / G \hbar}-1} .
$$

Replacing $\sigma \omega$ by $G \Delta \epsilon$, where $\Delta \epsilon$ is the energy of a shell, we arrive at the final result

$$
\left\langle\text { in }\left|\hat{N}_{\text {in }}\right| \text { in }\right\rangle=-\frac{\pi A R_{\mathrm{c}} \hbar(1+2 E)}{b \Delta \epsilon} \frac{1}{e^{-4 \pi A R_{c} \Delta \epsilon / \hbar}-1},
$$

from which we read off

$$
k_{\mathrm{B}} T_{\mathrm{c}}=-\frac{\hbar}{4 \pi A R_{\mathrm{c}}} .
$$


How can one interpret the prefactor in (45)? It should give the greybody factor, but its interpretation is complicated by the fact that the full normalization of our wave functionals is open. We thus do not address it any further.

Using (37), one can easily determine

$$
A=-\frac{3}{\Lambda\left(R_{\mathrm{c}}-R_{\mathrm{h}}\right)\left(R_{\mathrm{c}}-R_{\mathrm{n}}\right)}
$$

and thus finds

$$
k_{\mathrm{B}} T_{\mathrm{c}}=\frac{\hbar \Lambda\left(R_{\mathrm{c}}-R_{\mathrm{h}}\right)\left(R_{\mathrm{c}}-R_{\mathrm{n}}\right)}{12 \pi R_{\mathrm{c}}} .
$$

This result coincides with Equation (2.15b) in [14].

Interestingly, our calculation yields a Planck spectrum with a temperature that would be measured by a fictitious observer with $g_{T T}=1$. While such an observer could be easily realized in the Schwarzschild case (he would be situated at spatial infinity), this is not possible here. For the SdS case, the observer should be located between the horizons. Following [29], we shall consider the preferred observer who is stationary at a position $R_{\mathrm{o}}$ where the black-hole attraction and the cosmological expansion cancel each other; such an observer is thus unaccelerated and moves on a geodesic. We have 8

$$
R_{\mathrm{o}}=\left(\frac{3 G M}{\Lambda}\right)^{1 / 3}
$$

which leads to the modified Hawking temperature

$$
k_{\mathrm{B}} T_{\mathrm{c}}=\frac{1}{\sqrt{1-\left(9 G^{2} M^{2} \Lambda\right)^{1 / 3}}} \frac{\hbar \Lambda\left(R_{\mathrm{c}}-R_{\mathrm{h}}\right)\left(R_{\mathrm{c}}-R_{\mathrm{n}}\right)}{12 \pi R_{\mathrm{c}}} .
$$

This also agrees with the corresponding expression for the temperature given in Equation (17) in [15] where the Hawking temperature is calculated from a tunnelling picture.

A completely analogous calculation yields the Hawking temperature for the black-hole horizon. In fact, the corresponding overlap between $\Psi_{\mathrm{e}}^{-}$and $\Psi_{\mathrm{c}}^{+}$leads to the same expression (35) for $\beta$. In contrast to above, however, the near-horizon approximation is now performed for the black-hole horizon. Now, the coefficient $B$ in (37) enters. It is given by

$$
B=-\frac{3}{\Lambda\left(R_{\mathrm{h}}-R_{\mathrm{c}}\right)\left(R_{\mathrm{h}}-R_{\mathrm{n}}\right)},
$$

\footnotetext{
${ }^{8}$ For the above example of the supermassive black hole in the quasar OJ287, one gets $R_{\mathrm{o}} \approx 260 \mathrm{kpc}$, which corresponds to roughly five times our distance to the Large Magellanic Cloud!
} 
which follows from the expression for $A$ by interchanging $R_{\mathrm{c}}$ and $R_{\mathrm{h}}$. One thereby arrives at the expression

$$
k_{\mathrm{B}} T_{\mathrm{h}}=\frac{\hbar \Lambda\left(R_{\mathrm{c}}-R_{\mathrm{h}}\right)\left(R_{\mathrm{h}}-R_{\mathrm{n}}\right)}{12 \pi R_{\mathrm{h}}},
$$

which is equal to Eq. (2.15a) in [14]. This expression together with (47) are the main results of our paper, because they have been derived from candidates for exact quantum gravitational states. The temperature (50) will be modified by the same factor as in (49) if an observer is considered who follows the geodesic motion at $R_{\mathrm{o}}$.

The two temperatures (47) and (50) arise from the same expression (35) by two different near-horizon approximations. An exact evaluation of (35) would yield the sum of both temperatures plus interference terms between the two types of Hawking radiation. In the extremal case of the Nariai

metric, both temperatures are zero. Nevertheless, an exact evaluation of (35) could yield a small non-zero contribution which would be a genuine quantum gravitational effect.

To summarize, we have shown that solutions to the Wheeler-DeWitt equation and the diffeomorphism constraints contain information about the two Hawking temperatures from the black-hole and the cosmological horizon. An interesting open question is the calculation of the entropy for the Schwarzschild-de Sitter case through a counting of microstates. Such a derivation was presented for the BTZ black hole [30] and the AdS black hole [31. Whether it is also possible here is left for future publications.

Acknowledgements: We thank Rabin Banerjee, Max Dörner, T. P. Singh, Rakesh Tibrewala, and Cenalo Vaz for helpful discussions and critical comments. A.F. thanks the Tata Institute of Fundamental Research, Mumbai, for kind hospitality during her stay.

\section{References}

[1] J. Plebański and A. Krasiński, An Introduction to General Relativity and Cosmology (Cambridge University Press, Cambridge, 2006).

[2] C. Kiefer, Quantum Gravity, second edition (Oxford University Press, Oxford, 2007).

[3] C. Kiefer, Gen. Relativ. Gravit. 41, 877 (2009).

[4] K. V. Kuchař, Phys. Rev. D 50, 3961 (1994).

[5] J. Louko and S. N. Winters-Hilt, Phys. Rev. D 54, 2647 (1996).

[6] C. Vaz, L. Witten, and T. P. Singh, Phys. Rev. D 63, 104020 (2001). 
[7] C. Kiefer, J. Müller-Hill, and C. Vaz, Phys. Rev. D 73, 044025 (2006). Typos are corrected in arXiv:gr-qc/0512047v2.

[8] C. Kiefer, J. Müller-Hill, T. P. Singh, and C. Vaz, Phys. Rev. D 75, 124010 (2007).

[9] C. Vaz, S. Gutti, C. Kiefer, and T. P. Singh, Phys. Rev. D 76, 124021 (2007).

[10] G. Hinshaw et al., Astrophys. J. Suppl. 180, 225 (2009).

[11] C. Vaz, R. Tibrewala, and T. P. Singh, Phys. Rev. D 78, 024019 (2008).

[12] M. Bojowald, T. Harada, and R. Tibrewala, Phys. Rev. D 78, 064057 (2008); M. Bojowald, J. D. Reyes, and R. Tibrewala, Phys. Rev. D 80, 084002 (2009).

[13] K. H. Geyer, Astronomische Nachrichten 301, 135 (1980).

[14] G. W. Gibbons and S. W. Hawking, Phys. Rev. D 15, 2738 (1977).

[15] S. Stotyn, K. Schleich, and D. M. Witt, Class. Quantum Grav. 26, 065010 (2009).

[16] M. J. Valtonen et al., Nature 452, 851 (2008).

[17] A. Krasiński, Gen. Relativ. Gravit. 31, 945 (1999).

[18] K. V. Kuchař and C. Torre, Phys. Rev. D 43, 419 (1991); J. D. Brown and K. V. Kuchar, ibid. 51, 5600 (1995).

[19] G. Kunstatter and J. Louko, Phys. Rev. D 75, 024036 (2007).

[20] C. Kiefer and J. Louko, Ann. Phys. (Leipzig) 8, 67 (1999).

[21] D. Cangemi, R. Jackiw, and B. Zwiebach, Ann. Phys. (NY) 245, 408 (1996).

[22] T. Brotz and C. Kiefer, Phys. Rev. D 55, 2186 (1997).

[23] M. Ansorg and J. Hennig, Class. Quantum Grav. 25, 222001 (2008).

[24] K. Martel and E. Poisson, Am. J. Phys. 69, 476 (2001).

[25] C. Vaz, C. Kiefer, T. P. Singh, and L. Witten, Phys. Rev. D 67, 024014 (2003).

[26] R. Banerjee, C. Kiefer, and B. R. Majhi, in preparation.

[27] S. W. Hawking, Commun. Math. Phys. 43, 199 (1975). 
[28] I. S. Gradshteyn and I. M. Ryzhik, Table of Integrals, Series, and Products, 5th edition (Academic Press, San Diego, 1994), Sec. 3.381.5.

[29] R. Bousso and S. W. Hawking, Phys. Rev. D 54, 6312 (1996).

[30] C. Vaz, S. Gutti, C. Kiefer, T. P. Singh, and L. C. R. Wijewardhana, Phys. Rev. D 77, 064021 (2008).

[31] C. Vaz and L. C. R. Wijewardhana, Phys. Rev. D 79, 084014 (2009). 